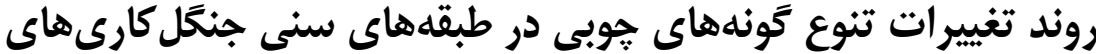

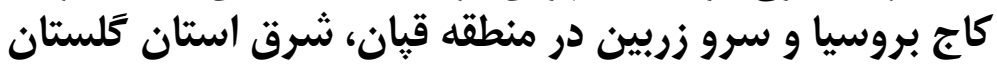

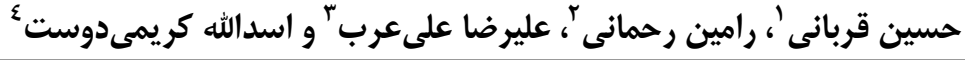

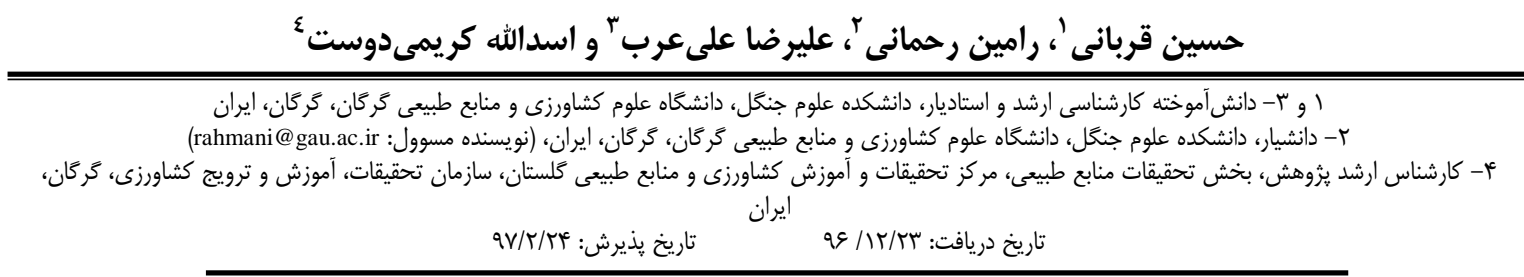

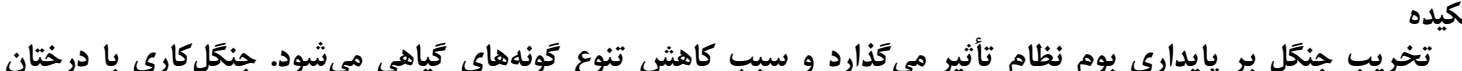

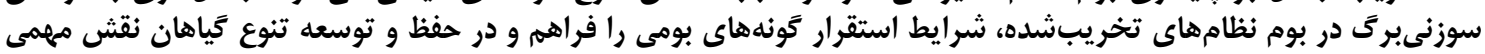

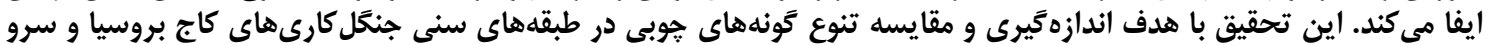

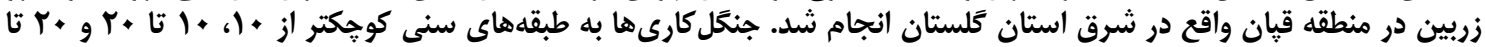

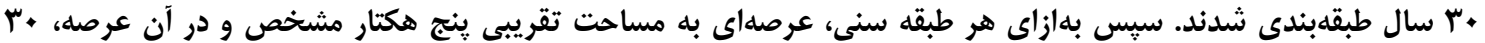

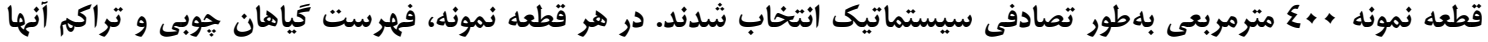

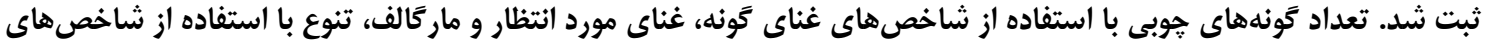

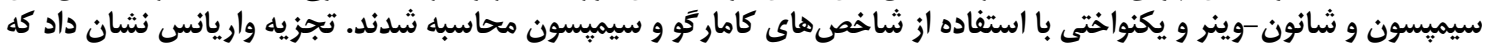

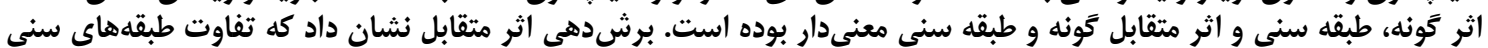

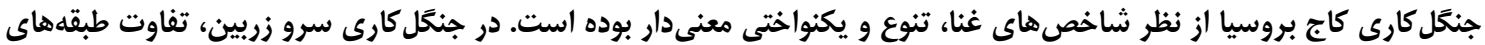

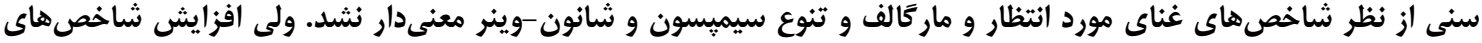

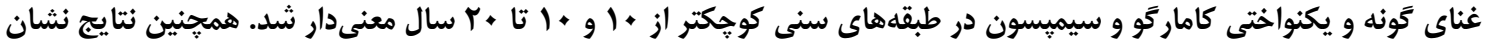

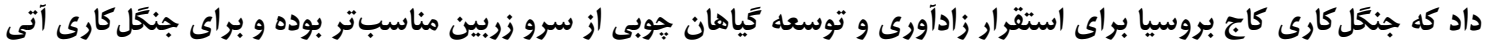

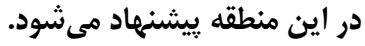

وازههاى كليدى: تنوع، جنكَل كارى، سوزنىبرتى، تونهاى جوبى، قِيان

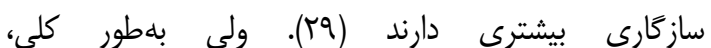

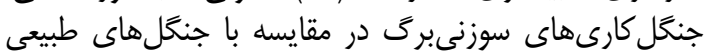

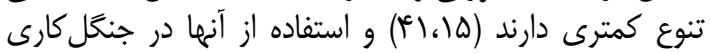

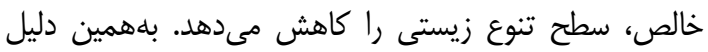

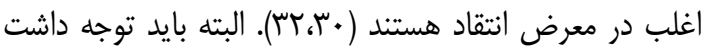

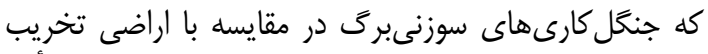

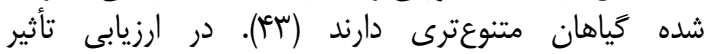

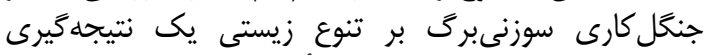

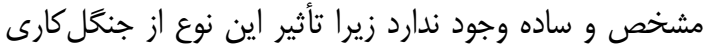

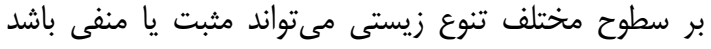

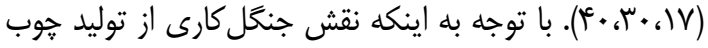

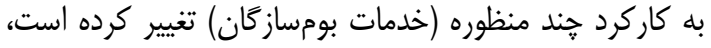

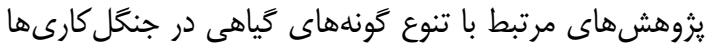

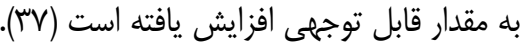

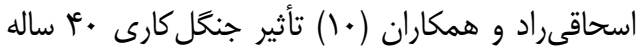

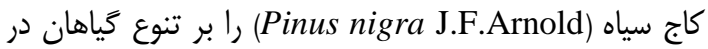

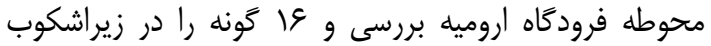

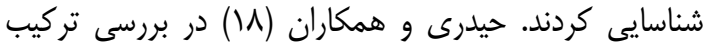

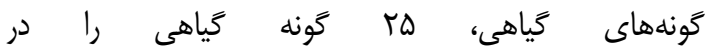

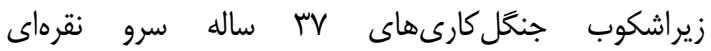
(Cupressus arizonica Greene) (Biota orientalis (L.) Endl.)
مقدمه

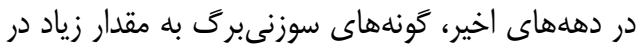

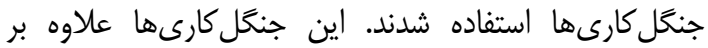

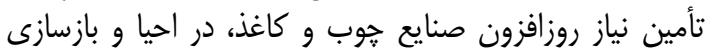

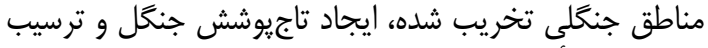

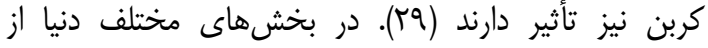

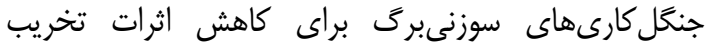

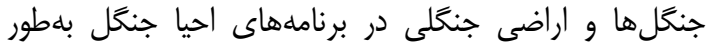

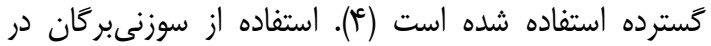

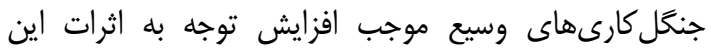

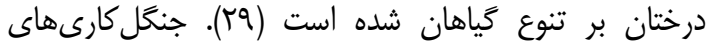

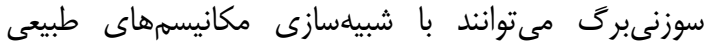

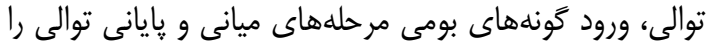

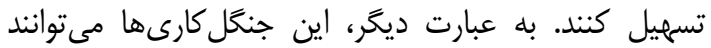

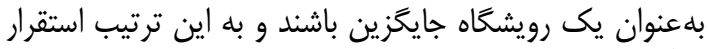

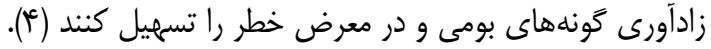

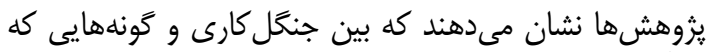

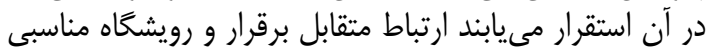

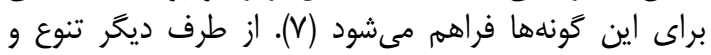

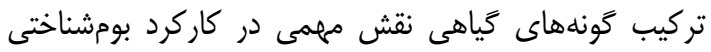

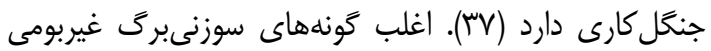

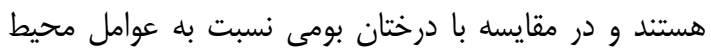


نتيجه كرفتند كه پايينترين مقدار غناى كونهاى و يكنواختى

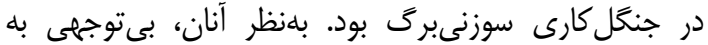

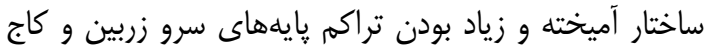

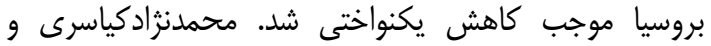

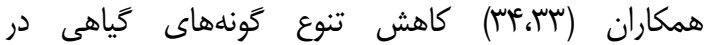

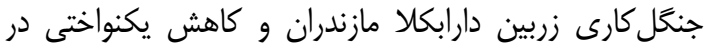

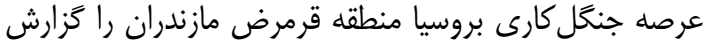

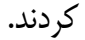

ايتو و همكاران (Tr) نتيجه كرفتند كه افزايش سن

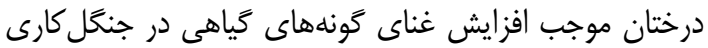

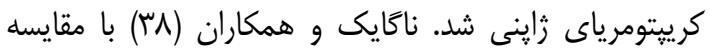

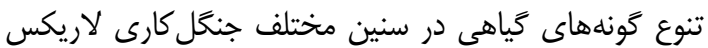

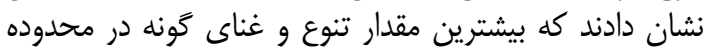

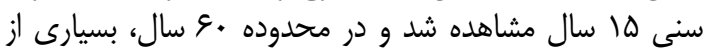

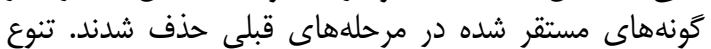

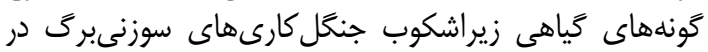

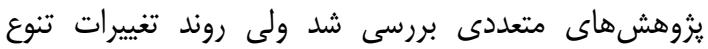

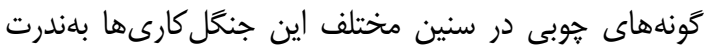

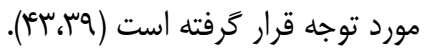

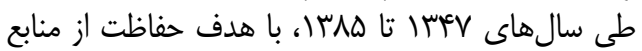

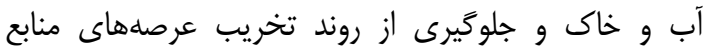

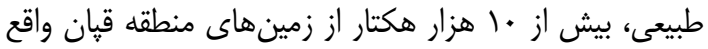

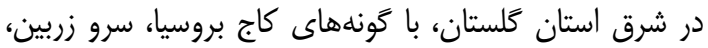

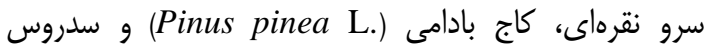
(Cedrus atlantica Manetti.)

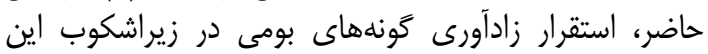

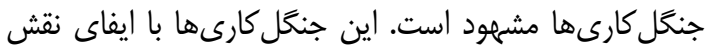

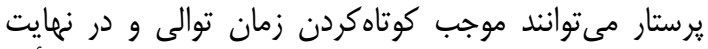

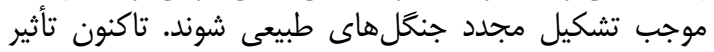

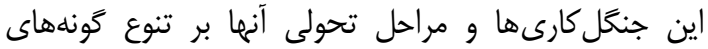

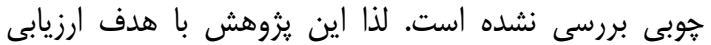

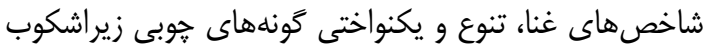

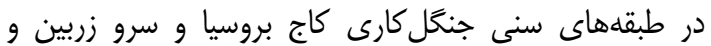
مقايسه تأثير طبقهاى سنى جنى بر اين شاخصها ئرا انجام شد.

\section{مواد و روشها منطقه مورد مطالعه}

منطقه مطالعهده در حوضه حورة آبخيز كركانرود و

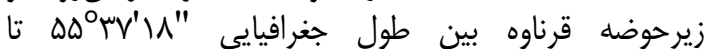
با

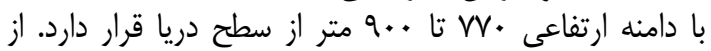

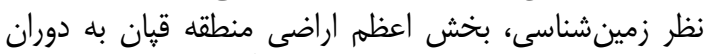

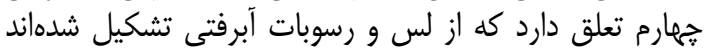

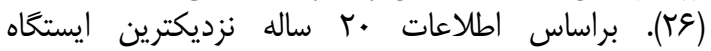

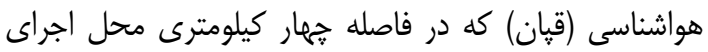
يزوهش قرار دارد، ميانكين بارندگى ساليانه

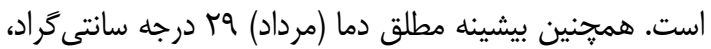

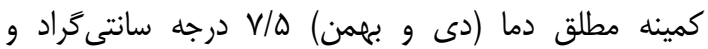

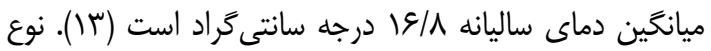

كردند. آنها نتيجه كَرفتند كه يوشش كَياهى زيراشكوب تحت

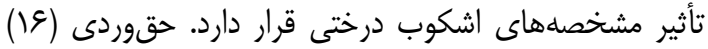

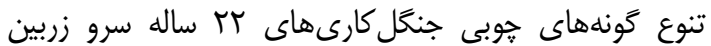
(Cupressus horizontalis Mill.)

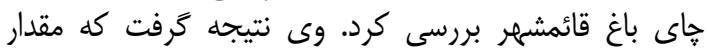

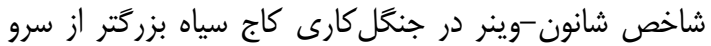

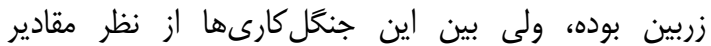

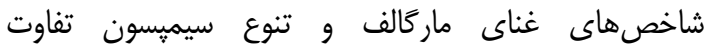

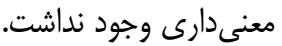

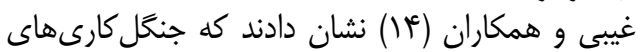
كاله توسكا ييلاقى (Alnus subcordata C.A.Mey)،

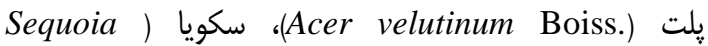
(sempervirens Endl.

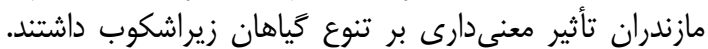

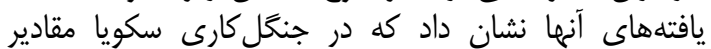

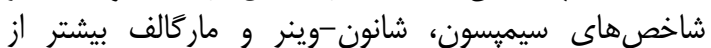

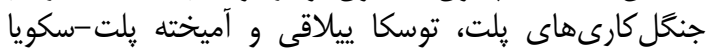

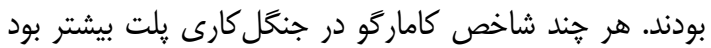

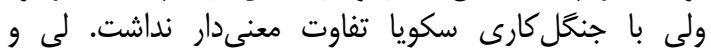

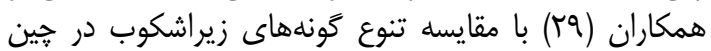
نشان دادند كه جنغكل كارى لاريكس (Larix kaempferi

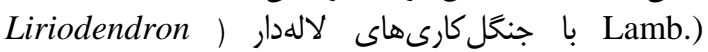
(chinense (Hemsl.) Sarg.

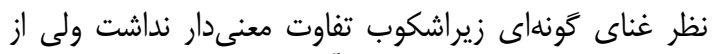

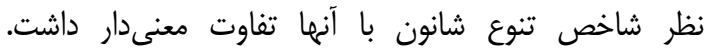

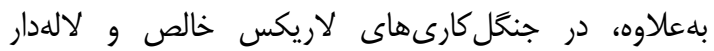

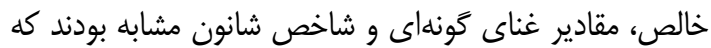

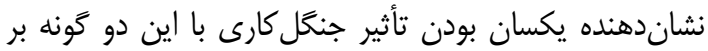

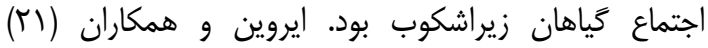

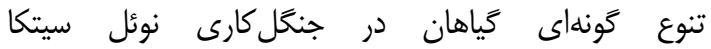
Picea abies و و نوئل (Picea sitchensis (Bong.) Carr.)

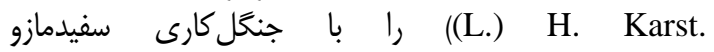
و و زبان كنجشك ارك (Quercus petraea (Matt.) Liebl.) (Fraxinus excelsior L.)

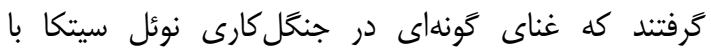

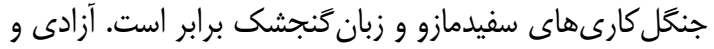

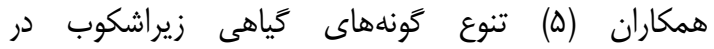

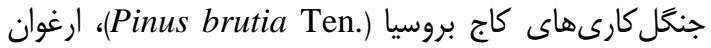

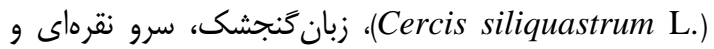

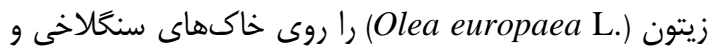

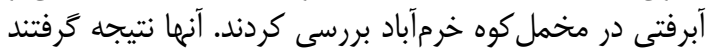

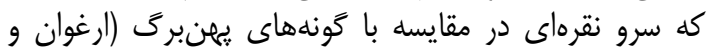

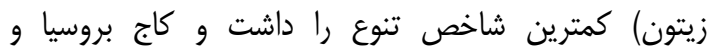

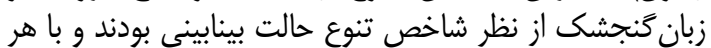

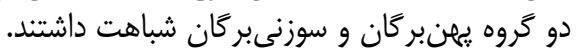

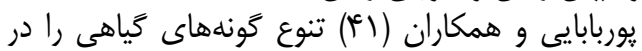

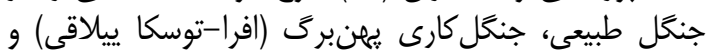

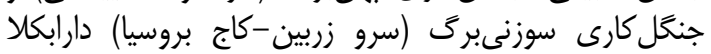

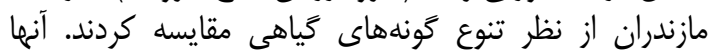


براى تمامى متغيرها معنىدار شد و به همين سبب مقايسه

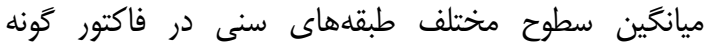

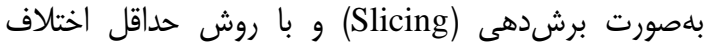

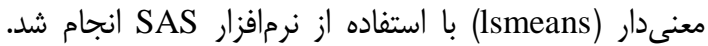

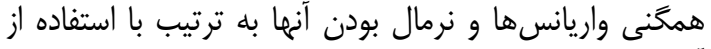

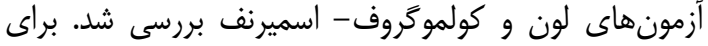

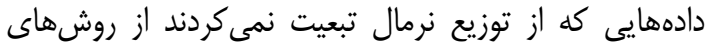

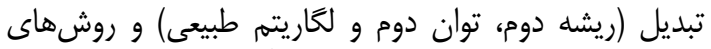

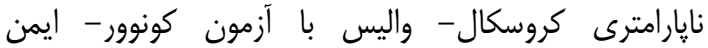
و من - ويتنى استفاده شد. (Conover-Iman)

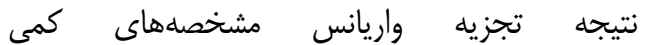

جنگل كارىهاى كاج بروسيا و سرو زربين نشان داد داد كه اثر

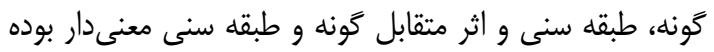

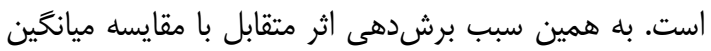

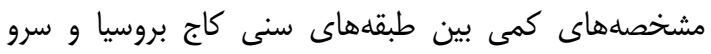

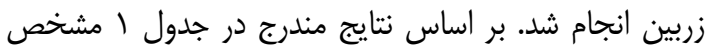
شد كه در جنگل كارى كاج سياه، با افزايش سن سن، تراكم

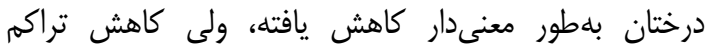

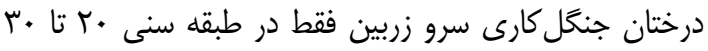

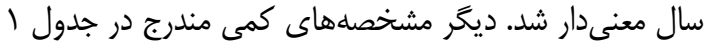

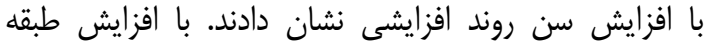

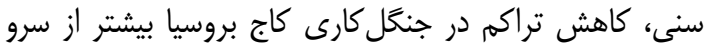

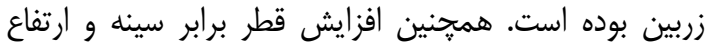

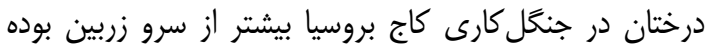

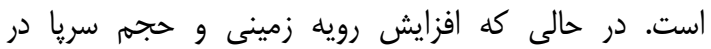

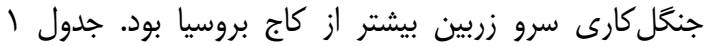

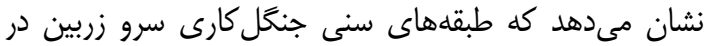

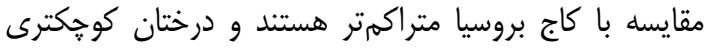

دارند.
اقليم براساس ضريب خشكى دومارتن نيمهخشك است. فصل خشك از خرداد آغاز مى شود و تا مهر إسى ادامه دارد.

روش تحقيق

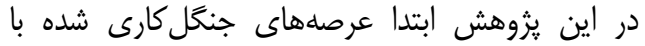

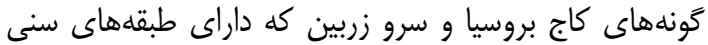

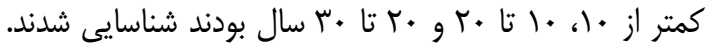

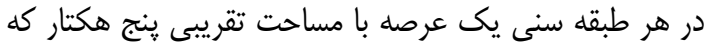

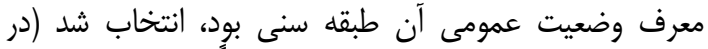

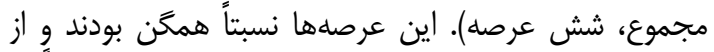

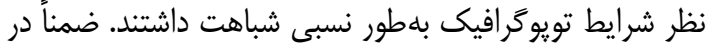

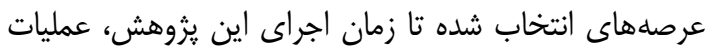

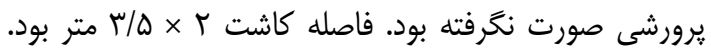

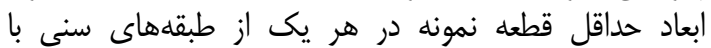

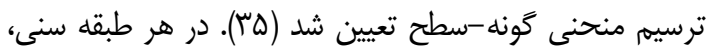

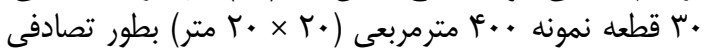

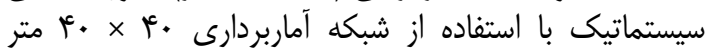

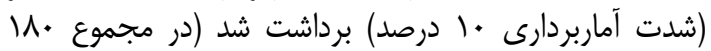

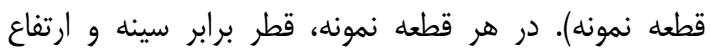

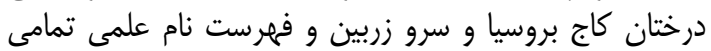

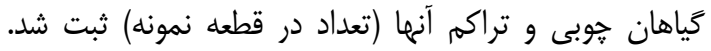

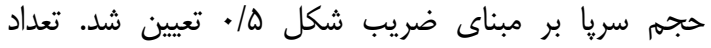

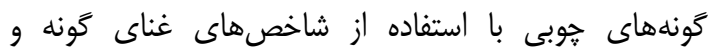

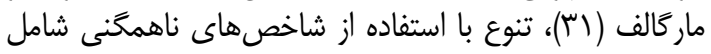

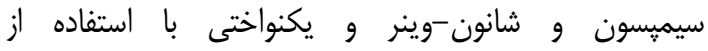

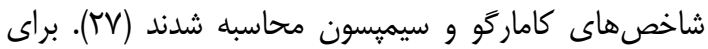

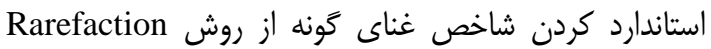

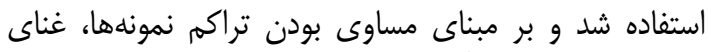

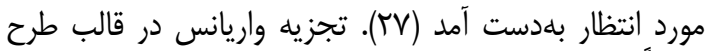

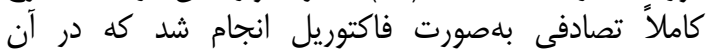

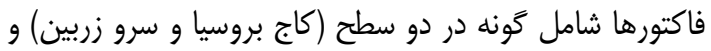

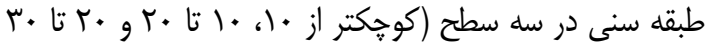

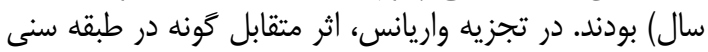

جدول ا- مقايسه ميانگين مشخصههاى كمى جنگَل كارىهاى كاج بروسيا و سرو زربين Table 1. Mean comparison of quantitative characteristics of Pinus brutia and Cupressus horizontalis plantations جنگل كارى سرو زربين جنگل كارى كاج بروسيا

\begin{tabular}{|c|c|c|c|c|c|c|}
\hline $\begin{array}{l}\dot{2} \\
\dot{2} \\
\dot{2} \\
3 \\
3\end{array}$ & $\begin{array}{l}\dot{3} \\
\dot{2} \\
\dot{3} \\
3\end{array}$ & $\begin{array}{l}\frac{3}{3} \\
\frac{3}{3} \\
\dot{3} \\
3 \\
3\end{array}$ & $\begin{array}{l}\dot{2} \\
\dot{3} \\
\dot{2} \\
3 \\
3\end{array}$ & $\begin{array}{l}\dot{1} \\
\dot{1} \\
\dot{3} \\
\stackrel{3}{3}\end{array}$ & $\begin{array}{l}\frac{y}{3} \\
\dddot{3} \\
\dot{3} \\
\stackrel{3}{3}\end{array}$ & ميانكَين مشخصههاى كمى \\
\hline$V 19^{B}$ & $119^{A}$ & $\Lambda \mathcal{A}{ }^{A}$ & $\mu \Lambda^{c}$ & $\Delta \mu^{b}$ & var ${ }^{a}$ & تراكم (تعداد در هكتار) \\
\hline$N / r^{A}$ & $|F /|^{B}$ & $\Delta / \Gamma^{C}$ & $r M / r^{\mathrm{a}}$ & $1 \Delta / \Delta^{b}$ & $8 / 0^{\mathrm{c}}$ & قطر برابر سينه (سانتىمتر) \\
\hline $1 . / v^{\mathrm{A}}$ & $V / \mu^{B}$ & $r / F^{C}$ & $\mid r / *^{\mathrm{a}}$ & $N N^{b}$ & $r / q^{c}$ & ارتفاع (متر) \\
\hline $19 / 4^{\mathrm{A}}$ & $\mid r / \kappa^{B}$ & $r /{ }^{C}$ & $\mid r / r^{\mathrm{a}}$ & $1 . / \kappa^{b}$ & $r / r^{c}$ & رويه زمينى (متر مربع در هكتار) \\
\hline$\Lambda \Delta / \Delta^{\mathrm{A}}$ & $F / \kappa^{B}$ & $r / r^{C}$ & $81 / r^{\mathrm{a}}$ & $r \Delta / \Delta^{b}$ & $r / V^{c}$ & حجم سريا (متر مكعب در هكتار) \\
\hline
\end{tabular}

جنگَل كارى، تغيير تراكم در طبقهاى سنى و حفظ بقا يا

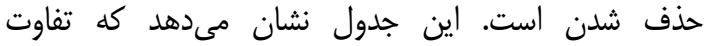

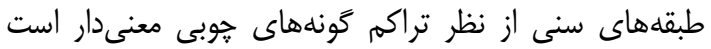

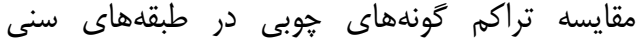

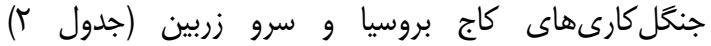

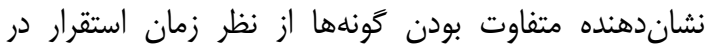


كه فقط در يك طبقه سنى حضور داشتند، انجاميذير نبود.

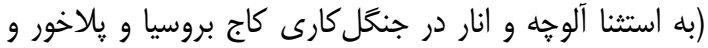

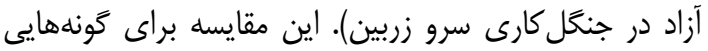

جدول r- تراكم (تعداد در هكتار) گونههاى جوبى در طبقهاى سنى جنگل كارىهاى كاج بروسيا و سرو زربين Table 2. Woody species density in age classes of Pinus brutia and Cupressus horizontalis plantations.

\begin{tabular}{|c|c|c|c|c|c|c|c|c|}
\hline \multirow[b]{2}{*}{ 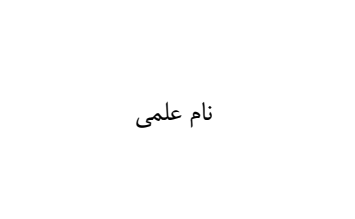 } & \multirow[b]{2}{*}{ 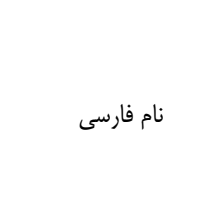 } & \multirow[b]{2}{*}{ 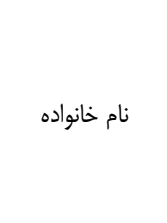 } & \multicolumn{3}{|c|}{ جنكل كارى سرو زربين } & \multicolumn{3}{|c|}{ جنكل كارى كاج بروسيا } \\
\hline & & & $\begin{array}{l}\dot{2} \\
\dot{3} \\
\dot{i} \\
3 \\
3\end{array}$ & $\begin{array}{l}\dot{5} \\
\dot{2} \\
\dot{2} \\
3 \\
3\end{array}$ & $\begin{array}{l}\frac{3}{3} \\
\overline{2} \\
\dot{3} \\
3 \\
3\end{array}$ & $\begin{array}{l}\dot{2} \\
\dot{2} \\
\dot{i} \\
3 \\
3\end{array}$ & $\begin{array}{l}\dot{5} \\
\dot{2} \\
\dot{2} \\
3 \\
\overrightarrow{3}\end{array}$ & $\begin{array}{l}\frac{y}{3} \\
\overline{3} \\
\dot{3} \\
\frac{3}{3}\end{array}$ \\
\hline Acer turkomanicum Pojark. & افرا تركمى & Aceraceae & 9 & - & - & $\Delta q$ & - & - \\
\hline Berberis integrrima Bge. & 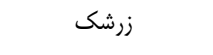 & Berberidaceae & - & - & rq & - & - & - \\
\hline Celtis caucasica Willd. & 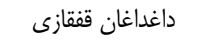 & Ulmaceae & 9 & - & - & rq & - & - \\
\hline Colutea porphyrogramma Rech .f. & دغدغك ارغوانى & Fabaceae & - & - & r & - & - & - \\
\hline Cornus australis C. A.Mey. & 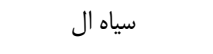 & Cornaceae & - & - & - & (1) & - & - \\
\hline Crataegus microphylla C.Koch. & 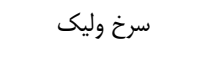 & Rosaceae & $v r^{A}$ & $18^{\mathrm{B}}$ & - & $v \notin \varepsilon^{a}$ & $9 \Lambda^{\mathrm{b}}$ & $r \cdot c$ \\
\hline Ephedra distachya $\mathrm{L}$. & ارمك دورديفى & Ephedraceae & - & $\wedge$ & - & - & - & - \\
\hline Lonicera korolkovii Stapf. & 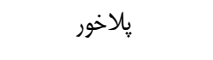 & Caprifoliaceae & - & $v \Lambda^{A}$ & $r q^{\mathrm{A}}$ & $i v \Lambda^{\mathrm{a}}$ & $\Delta F^{b}$ & - \\
\hline Lycium kopetdaghi Pojark. & 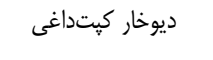 & Solanaceae & - & - & 19 & - & - & - \\
\hline Paliurus spina-christi Miller. & 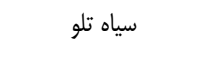 & Rhamnaceae & $r \Delta \Delta^{\mathrm{A}}$ & $r_{R} \mu^{A}$ & B & $r \Delta \Lambda^{a}$ & $|g|^{b}$ & $\Delta \Lambda^{c}$ \\
\hline Prosopis farcta (Banks \& Soland.) & كمورك & Fabaceae & - & - & lQV & - & - & - \\
\hline Prunus divaricate Ledeb. & آلوخه & Rosaceae & $\mu_{\Lambda}^{A}$ & $\wedge^{\mathrm{B}}$ & - & $v r^{\mathrm{a}}$ & $\Delta q^{\mathrm{a}}$ & - \\
\hline Punica granatum $\mathrm{L}$. & انار & Punicaceae & $v^{\mathrm{AB}}$ & rma & $q^{\mathrm{B}}$ & ()$^{\mathrm{a}}$ & $r I^{\mathrm{a}}$ & - \\
\hline Rhamnus sintenisii Rech. f. & 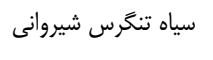 & Rhamnaceae & - & $\Delta \mathrm{H}^{\mathrm{A}}$ & $r \cdot{ }^{B}$ & $M^{b}$ & (سוץ & $r^{c}$ \\
\hline Rosa iberica Stev. & رز & Rosaceae & - & - & - & fir & - & - \\
\hline Ulmus minor Miller. & 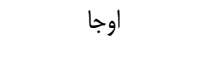 & Ulmaceae & - & - & - & $\Delta$ & - & - \\
\hline Zelkova carpinifolia Pall. & آزاد & Ulmaceae & $i v^{B}$ & $\pi r^{A}$ & $1^{\mathrm{B}}$ & 109 & - & - \\
\hline
\end{tabular}

حروف انغليسى نشاندهنده تفاوت معنىدار در سطح احتمال ينج درصد (كروسكال-واليس با أزمون كونور و من -ويتنى) بين طبقهاى سنى جنگل كارىهاى كاج بروسيا (حروف كوحى) و سرو زربين (حروف بزرك) هستند.

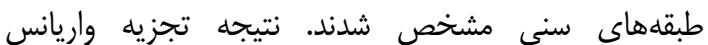

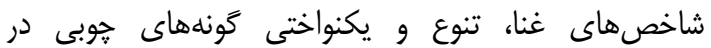

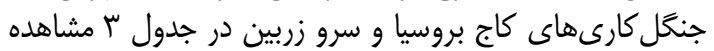

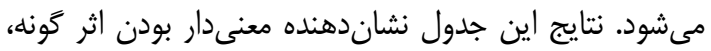

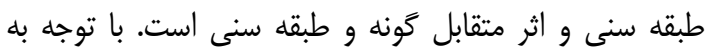

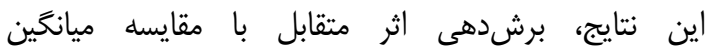

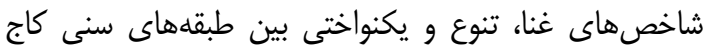

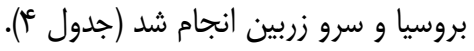

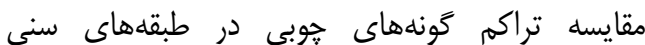

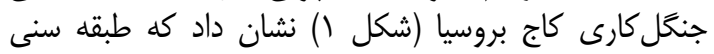

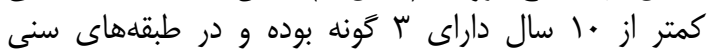

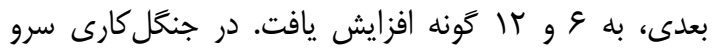

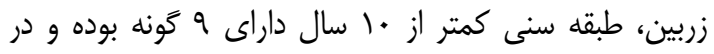

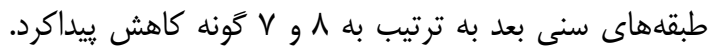

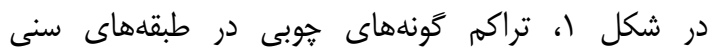

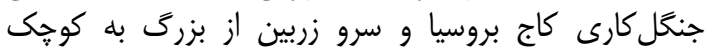

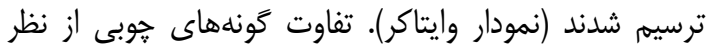

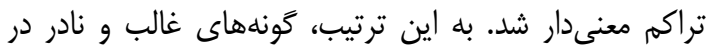



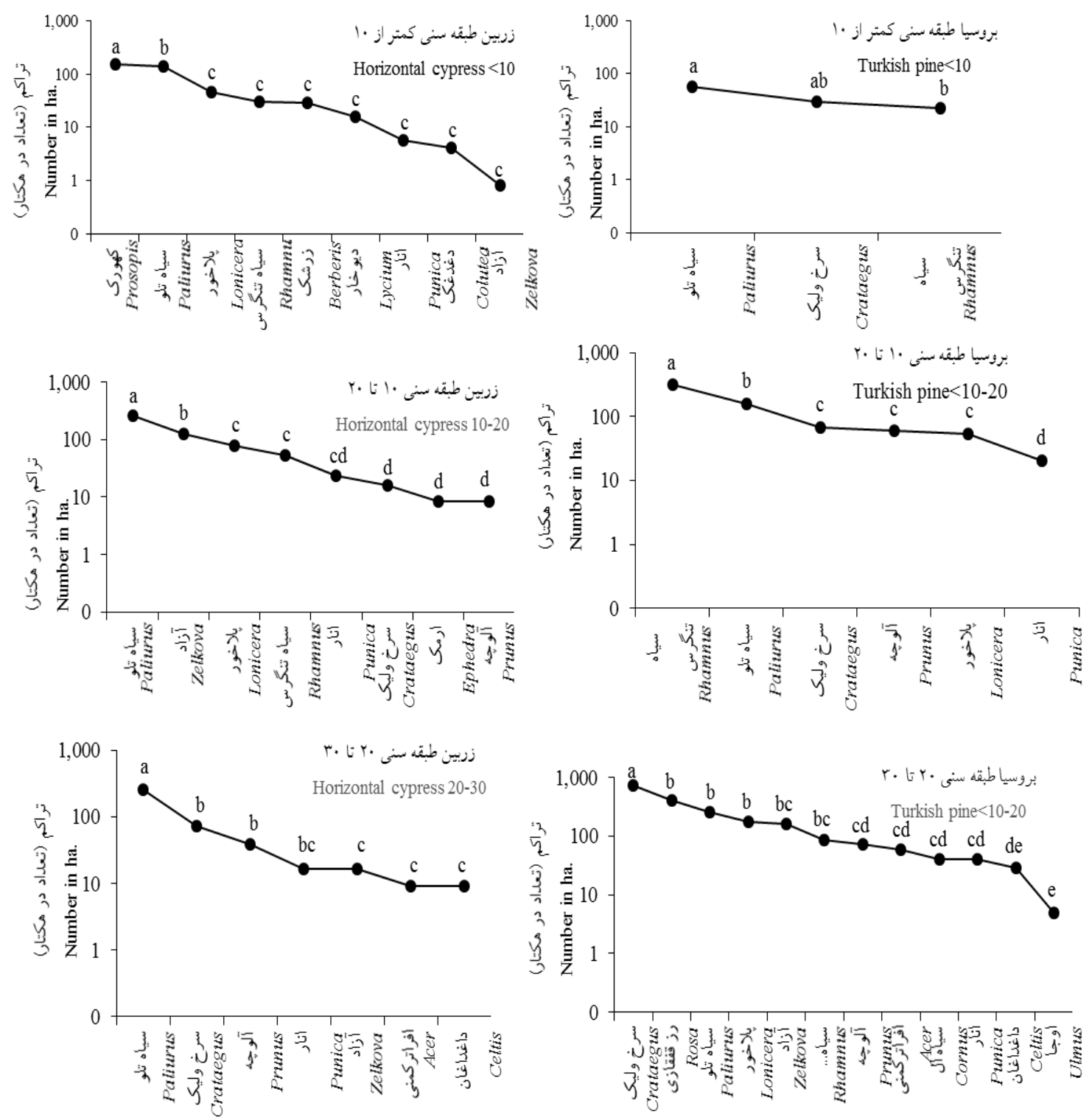

حروف انغليسى نشاندهنده تفاوت معنى دار بين كونهها هستند (آزمون كروسكال-واليس، در سطح احتمال ينج درصد).

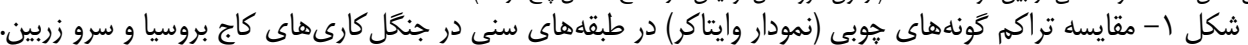

Figure1. Comparison of woody species density (Whittaker diagram) in age classes in Pinus brutia and Cupressus horizontalis plantations.

جدول س- تجزيه واريانس شاخصهاى غنا، تنوع و يكنواختى گونههاى جوبى در طبقهاى سنى جنگل كارىهاى كاج بروسيا و سرو زربين. Table 3. ANOVA results of richness, diversity and evenness indices of woody species in age classes in Pinus brutia and Cupressus horizontalis plantations

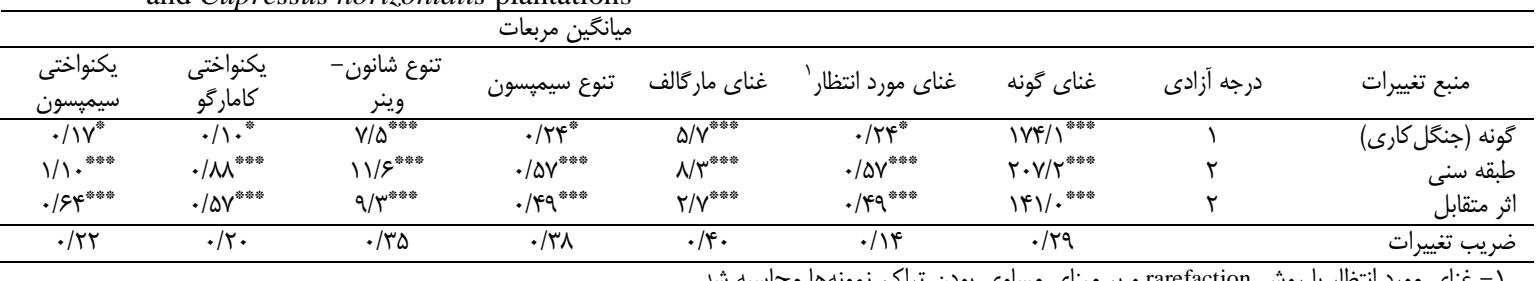

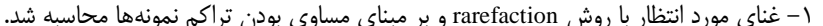

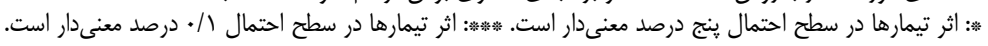


و شاخصهاى تنوع سيميسون و شانون-وينر تفاوت معنىدار

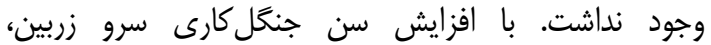

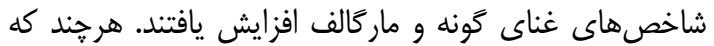

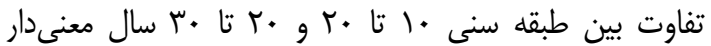

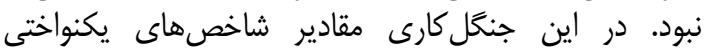

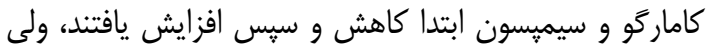

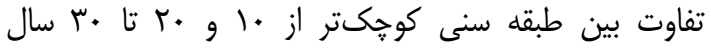

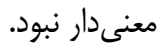

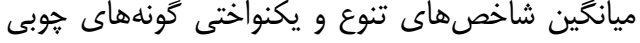

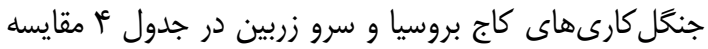

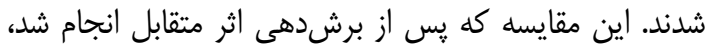

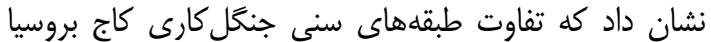

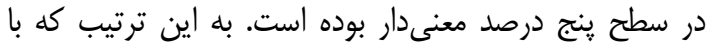

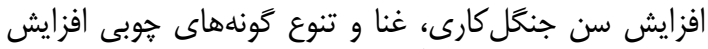

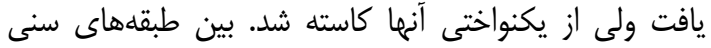
جنگَل كارى سرو زربين از نظر شاخصهاى غناى غناى مورد انتظار

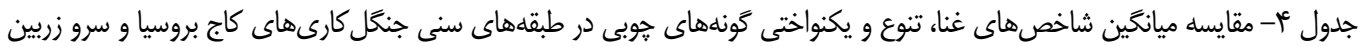
Table 4. Mean comparison of richness, diversity and evenness indices of woody species in age classes in Pinus brutia and Cupressus horizontalis plantations

\begin{tabular}{|c|c|c|c|c|c|c|}
\hline \multicolumn{3}{|c|}{ جنغَل كارى سرو زربين } & \multicolumn{3}{|c|}{ جنكَل كارى كاج بروسيا } & \multirow[b]{2}{*}{ شاخصها } \\
\hline $\begin{array}{l}i \\
i \\
i \\
3\end{array}$ & $\underset{\dot{2}}{\dot{2}}$ & $\begin{array}{l}\frac{y}{3} \\
\stackrel{3}{-3} \\
\vdots\end{array}$ & $\begin{array}{l}\dot{2} \\
\dot{2} \\
i \\
5\end{array}$ & $\stackrel{\dot{5}}{\dot{2}} \underset{3}{3}$ & $\begin{array}{l}\frac{y}{3} \\
\frac{3}{-}\end{array}$ & \\
\hline A & $r / \Delta \& V^{A}$ & B/Y B B & $\Lambda / V \mu \mu^{a}$ & $c / q \ldots{ }^{b}$ & $r / \ldots^{c}$ & غناى كَونه \\
\hline $1 / \Delta \mu^{A}$ & V/DFWA & $1 / \Delta r \Lambda^{A}$ & $1 / V r \Lambda^{a}$ & $1 / \mathrm{V} \cdot 9^{\mathrm{a}}$ & $1 / r^{\prime b}$ & غناى مورد انتظار' \\
\hline.$/ Q 9 V^{A}$ & $\cdot|\wedge \varepsilon|^{A}$ & $. / 9 \times 9 B$ & $1 / v \Delta a^{a}$ & $1 / 198^{b}$ & $\cdot / \Delta 99^{c}$ & غناى ماركالف \\
\hline$\cdot / \Delta r r^{A}$ & - $/ \Delta F{ }^{A} A$ & $\cdot / \Delta T \Lambda^{A}$ & $\cdot / V Y \Lambda^{a}$ & $\cdot / V \cdot 9^{\mathrm{a}}$ & $\cdot / q^{\prime b}$ & تنوع سيميسون \\
\hline $1 / r \mid F^{A}$ & $1 / r F \theta^{A}$ & $1 / 199^{\mathrm{A}}$ & $r / r r \omega^{a}$ & $1 / 199^{b}$ & $\cdot / V Y Y^{c}$ & تنوع شانون - وينر \\
\hline$\cdot / V^{r} \varphi^{A}$ &.$|8| 8^{\mathrm{B}}$ & $\cdot / W V \varepsilon^{A}$ &.$/ 4 \& V^{c}$ &.$/ 94 \varepsilon^{b}$ & $\cdot / \Lambda \gamma^{\mathrm{u}^{\mathrm{a}}}$ & يكنواختى كاماركً \\
\hline$\cdot / N^{e} q^{A}$ & $\cdot \mid a r \Lambda^{B}$ & $\cdot / \Lambda r \cdot{ }^{A}$ &.$/ 4 F 9^{c}$ & $\cdot / 9 \vee \Delta^{\mathrm{b}}$ & $\cdot / \wedge 90^{a}$ & يكنواختى سيميسون \\
\hline
\end{tabular}

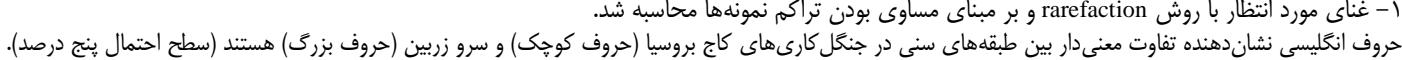

گَونه و ماركالف در طبقه سنى كمتر از • مال سال داراى كمترين

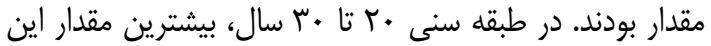

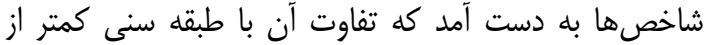

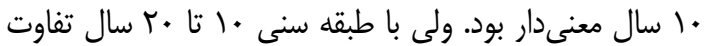

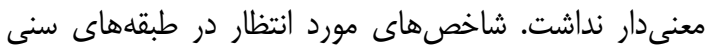

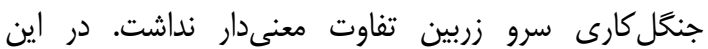

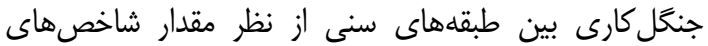

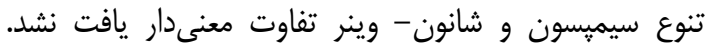

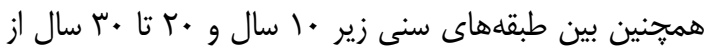

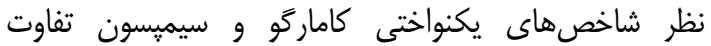

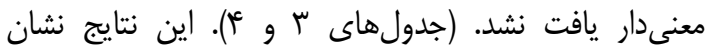

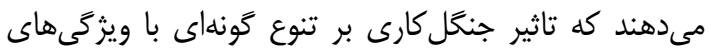

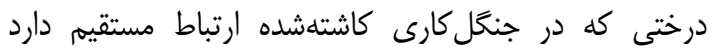
(rV)

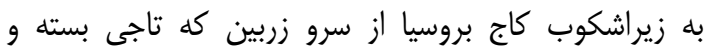

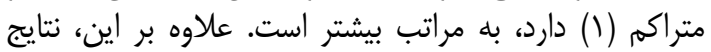

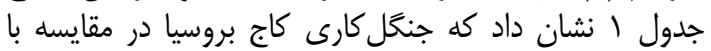

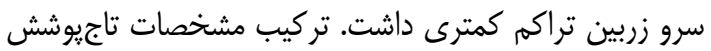

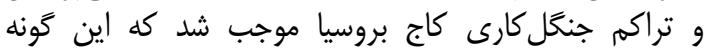

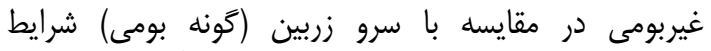

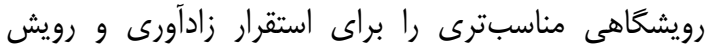

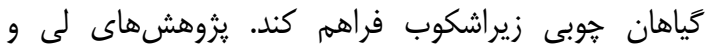

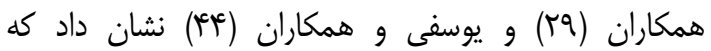

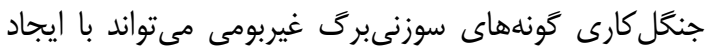

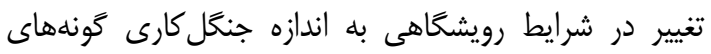

در يزوهش حاضر تراكم و تنوع گونههاى جوبى در

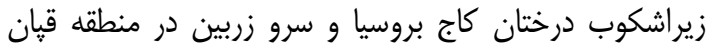

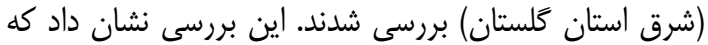

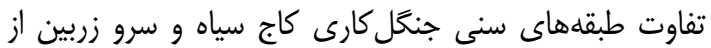

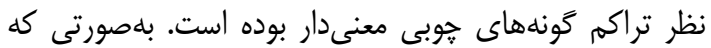

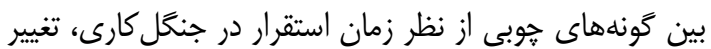

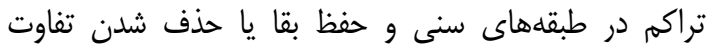

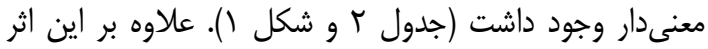

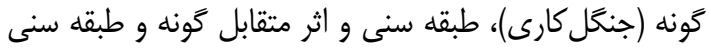

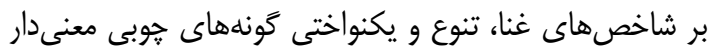

در جنگل كارى كاج بروسيا، شاخصهاى غناى گَّنه،

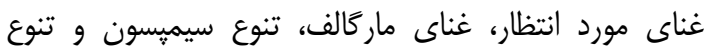

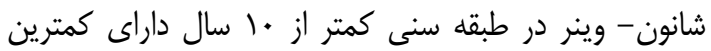

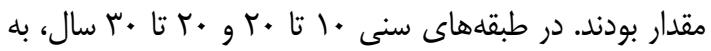

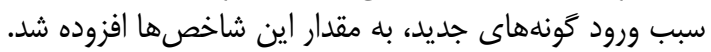

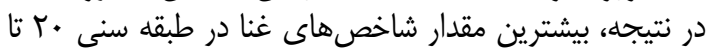

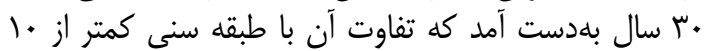

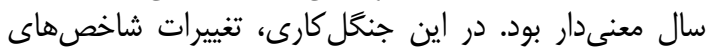

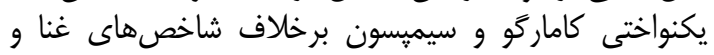

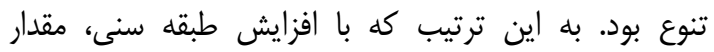

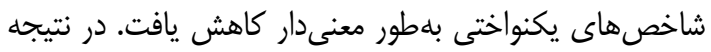

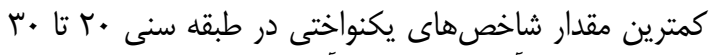

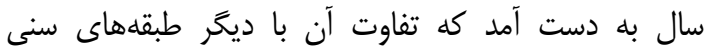

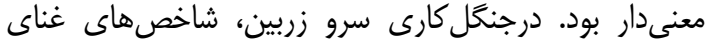




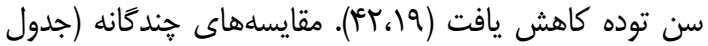

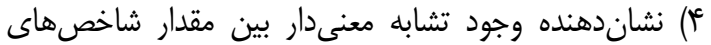

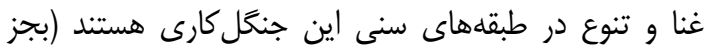

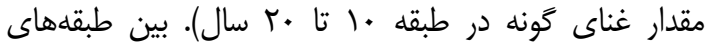

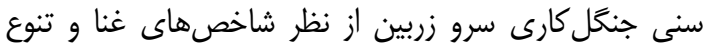

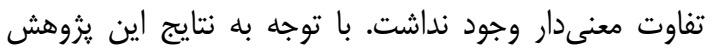

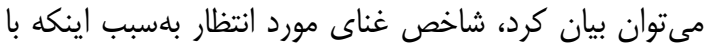

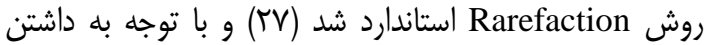
كوجكترين مقدار ضريب تغييرات، براى مقايسه تأثير طبقهاى سنى بر غنا و تنوع كياهان جوبى زئى زيراشكوب

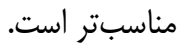
افزايش شاخصهاى غنا و تنوع در جنكَلكارى كاج

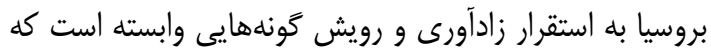

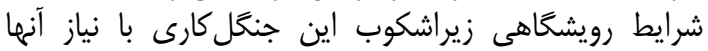

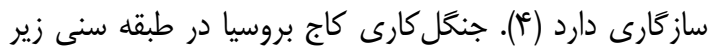

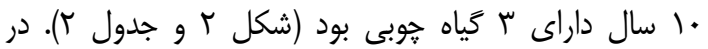

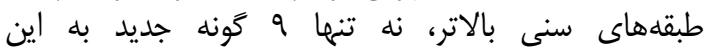

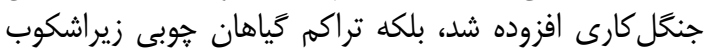

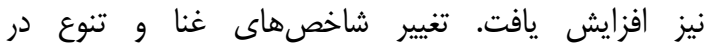

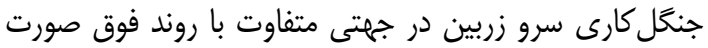

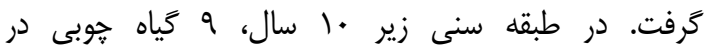

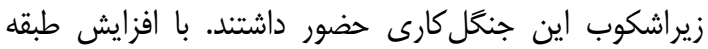

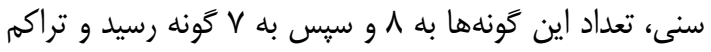

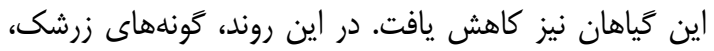

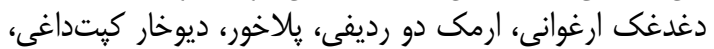

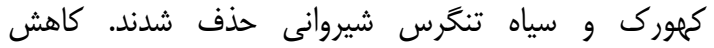

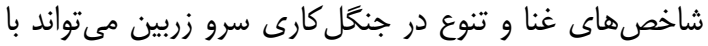

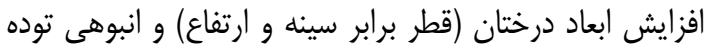

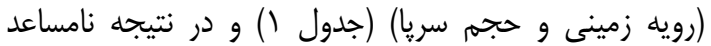

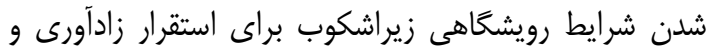

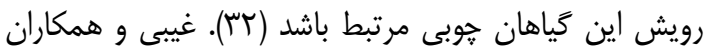

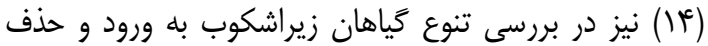
كونههاى كياهى در طول زمان اشاره كردنئ

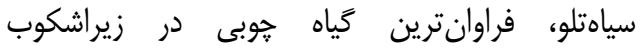

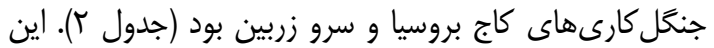

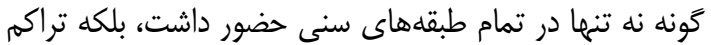

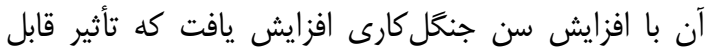

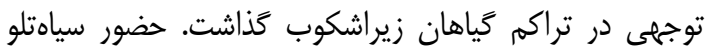

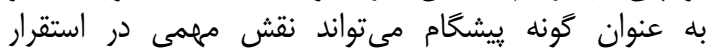

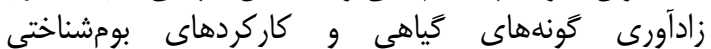

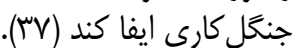

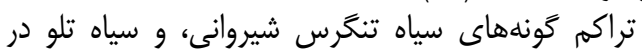

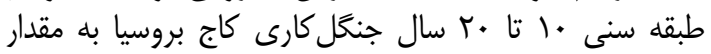

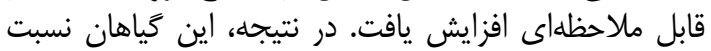

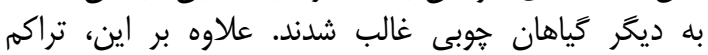
كونهاى رز قفقازى، سرخ وليك، آزاد، سياه تلو تلو و و سياه

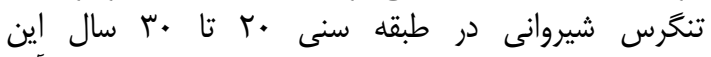

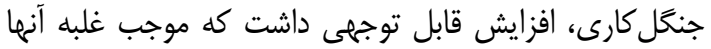
بر ديخر كياهان جوبى شد. افزايش تراكم اين كياهان إنان و غالب
بومى در افزايش تنوع كَونهاى بومى زيراشكوب تأثير داشته

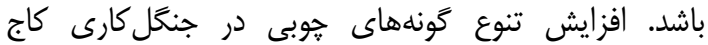

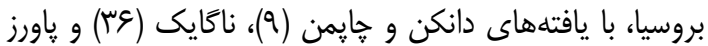

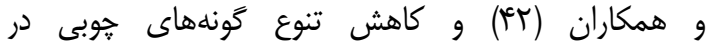

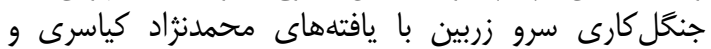

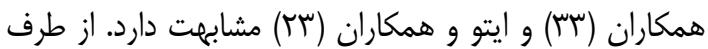

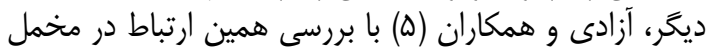

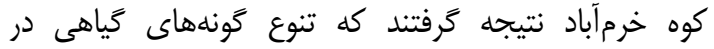

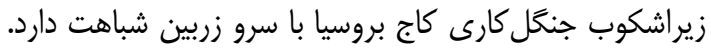

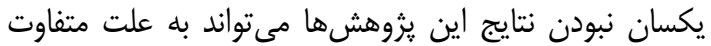

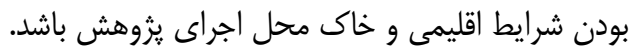

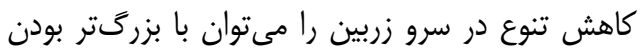

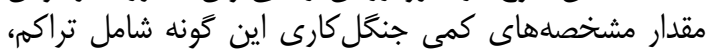

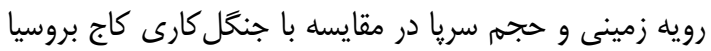

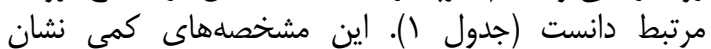

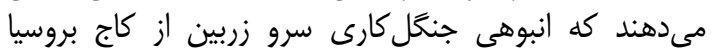

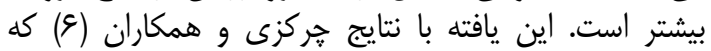

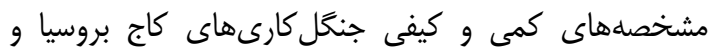
سرو زربين را در شرق استان كلستان (راميان) بررسى كراج كردند

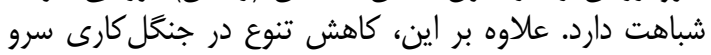

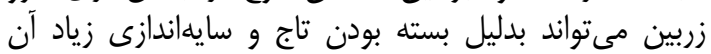

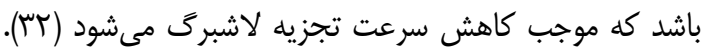

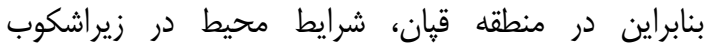

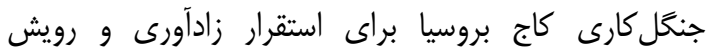

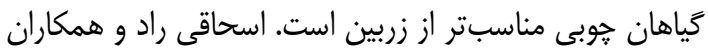

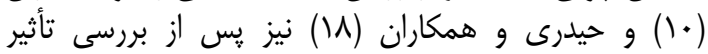

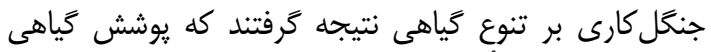

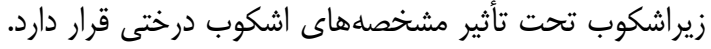

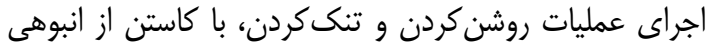

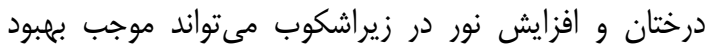
استقرار كياهان جوبى و افزايش تنوع آنها در جنكل كاري شوان شود

برشدهى اثر متقابل نشان داد كه تفاوت طبقههاى

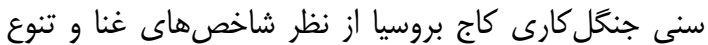

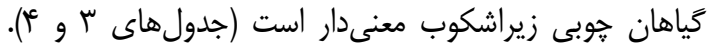

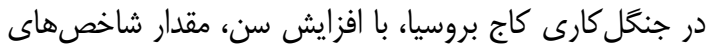

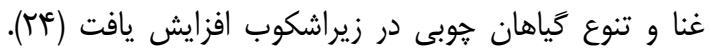

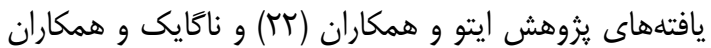

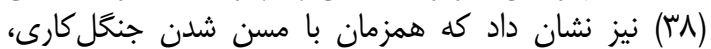

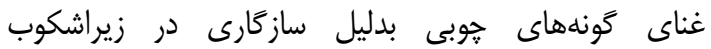

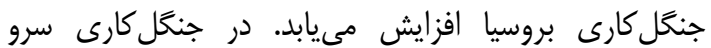

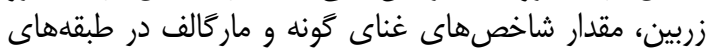

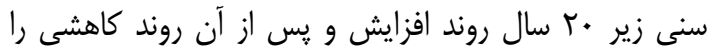

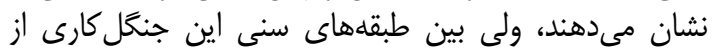

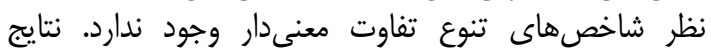

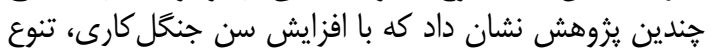

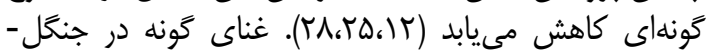

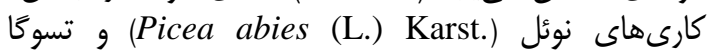
همزمان با افزايش (Tsuga heterophylla (Raf.) Sarg.) 
جنكل كارى كاج بروسيا، يكنواختى گياهان خوبى زيراشكوب

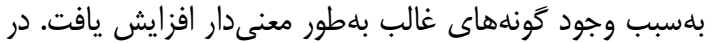

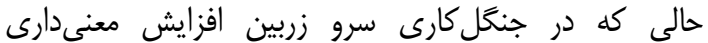

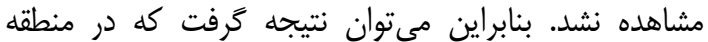

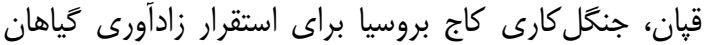

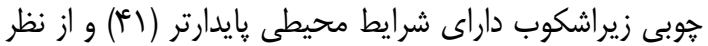

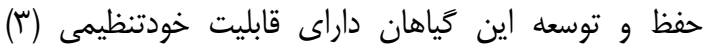

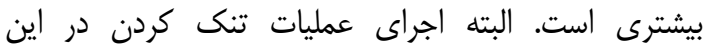

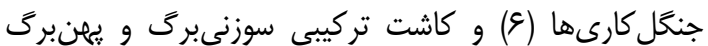

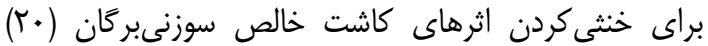

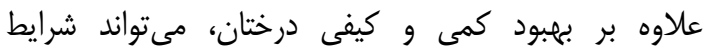

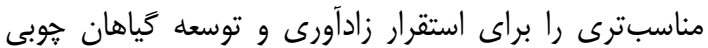

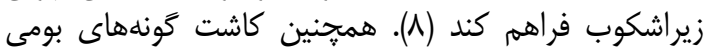

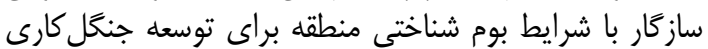

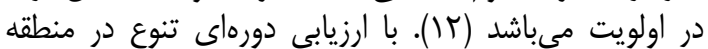

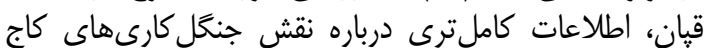

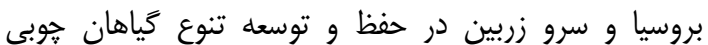
زيراشكوب بهدست خواهد آمد.
شدن آنها در اجتماع كَياهان خوبى مهمترين دليل كاهش

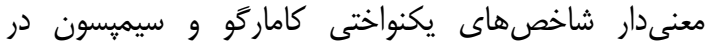

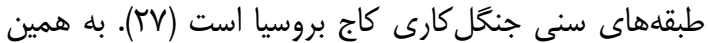

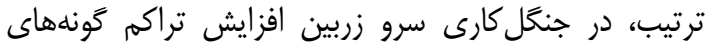

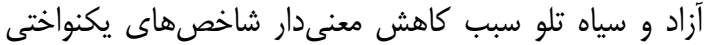

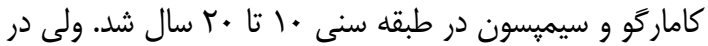

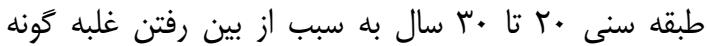

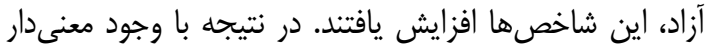

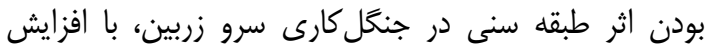
سن، شاخصهاى يكنواختى ابتدا افزايش و سيس رِّ كاهش يافتند. بر اساس يافتهاى يثزوهش حاضر مشخص شئ كار كه

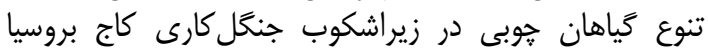

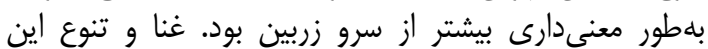

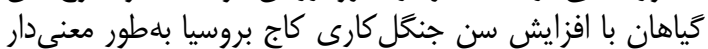

افزايش يافت. ولى در جنكا كارى سرو زربين، اين افزايش

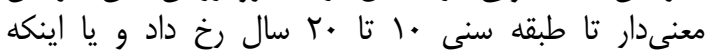
افزايش معنىدارى مشاهده نشد. علاوه بر اين، با مسن شاد شن إن إنكا

1. Abbasi, H. 1990. Fast-growing trees. Gorgan Jahad-e-Daneshghahi press, 190 pp (In Persian).

2. Afforestation and Parks Office. 1995. Coniferous plantations in the North of Iran. Forests, Range and Watershed Management Organization, 113 pp (In Persian).

3. Ardakani, M.R. 2003. Ecology. Tehran University press, $331 \mathrm{pp}$ (In Persian).

4. Avendaño-Yáñez, M.L., L.R. Sánchez-Velásquez, J.A. Meave and M.R. Pineda-López. 2016. Can Pinus plantations facilitate reintroduction of endangered cloud forest species? Landscape and Ecological Engineering, 12(1): 99-104.

5. Azadi, A., S.M. Hojati, H. Jalilvand and H. Naghavi. 2014. Investigation on soil carbon sequestration and understory biodiversity of hard wood and soft wood plantations of Khoramabad city (Makhmalkoh site). Iranian Journal of Forest and Poplar Research, 21(4): 702-715 (In Persian).

6. Charkazi, A., M. Amiri, H. Ravanbakhsh and M. Moghadasi. 2016. Examination of quantitative and qualitative characteristics of Cupressus sempervirens var. horizontalis and Pinus brutia in plantation forests in the Ramian, Golestan province. Journal of Wood and Forest Science and Technology, 23(4): 1-21 (In Persian).

7. Coote, L., L.J. French, K.M. Moore, F.J.G. Mitchell and D.L. Kelly. 2012. Can plantation forests support plant species and communities of semi-natural woodland? Forest Ecology and Management, 283: 86-95.

8. Cusack, D. and F. Montagnini. 2004. The role of native species plantations in recovery of understory woody diversity in degraded pasturelands of Costa Rica. Forest Ecology and Management, 188: 1-15.

9. Duncan, R.S. and C.A. Chapman. 2003. Consequences of plantation harvest during tropical forest restoration in Uganda. Forest Ecology and Management, 173: 235-250.

10. Eshaghi Rad, J., P. Ghaffarnejad and A. Banedg Shafiee. 2014. Quantitative evaluation of Pinus nigra plantation and its effect on plant diversity and soil chemical properties of rangeland ecosystems (Case study: Urmia airport plantation). Iranian Journal of Forest, 6(4): 471-482 (In Persian).

11. Fazlolahi, M., A. Najafi, A. Soleimani and A. Sepahvand. 2014. Selection of the Most Suitable Species in Order to Forestation in Southern Zagros Forests using AHP and TOPSIS Techniques. Journal of Ecology of Iranian Forest, 2(4): 45-55 (In Persian).

12. Gara, R. and S. Healey. 2003. The effect of a teak (Tectonia grandis) plantation on the establishment of native species in an abandoned pasture in Costa Rica. Forest Ecology and Management, 176: 497507.

13. GDMG. (General Directorate of Meteorology Golestan Province). 2013. Twenty years statistics of Ghapan station (In Persian).

14. Gheibi, F., M. Akbarinia and Y. Kooch. 2015. Effect of Alnus subcordata, Acer insigne and Sequoia sempervirens plantations on plant diversity in Hyrcanian forest of Iran. Biodiversitas, 16(1): 10-15.

15. Gerstner, K., C.F. Dormann, A. Stein, A.M. Manceur and R. Seppelt. 2014. Effects of land use on plant diversity: a global meta-analysis. Journal of Applied Ecology, 51: 1690-1700.

16. Haghverdi, K. 2015. Influence of endemic and exotic afforestation (Chai Bagh district of Qaemshahr) on biodiversity of plant species and woody regeneration. Journal of Plant Researches, 28(3): 522-534 (In Persian).

17. Hartley, M.J. 2002. Rational and methods for conserving biodiversity in plantation. Forest Ecology and Management, 155: 81-95. 
18. Heydari, M., N. Shabanian, M. Zeinivand Zadeh and J. Mirzaei. 2015. Analysis of understory plant composition in hardwoods and conifers plantations and their relation to soil characteristics. Forest Research and Development, 1(2): 155-166 (In Persian).

19. Hill, M.O. and E.W. Jones. 1978. Vegetation changes resulting from afforestation of rough grazings in Caeo forest, South Wales. Journal of Ecology, 66: 433-456.

20. Hosseini, S. and V. Hosseini. 2014. Effect of Reforestation with Pinus nigra Arnold, Pinus eldarica Medw. and Cupressus arizonica Greene Species on some Properties of Soil (Case Study: Garan region, Marivan). Journal of Ecology of Iranian Forest, 2(4): 37-44 (In Persian).

21. Irwin, S., S.M. Pedley, L. Coote, A.C. Dietzsch, M.W. Wilson, A. Oxbrough, O. Sweeney, K.M. Moore, R. Martin, D.L. Kelly, F.J.G. Mitchell, T.C. Kelly and J. O'Halloran. 2014. The value of plantation forests for plant, invertebrate and bird diversity and the potential for cross-taxon surrogacy. Biodiversity and Conservation, 23(3): 697-714.

22. Ito, S., M. Nakagawa, G.P. Buckley and K. Nogami. 2003. Species richness in sugi (Cryptomeria japonica D. Don) plantations in southeastern Kyushu, Japan. Journal of Forest Research, 8: 49-57.

23. Ito, S., R. Nakayama and G.P. Buckley. 2004. Effects of previous land-use on plant species diversity in semi natural and plantation forests in warm temperate region in southern Kyushu, Japan. Forest Ecology and Management, 196: 213-235.

24. Johansson, O., C. Fries, B. Pettersson and P. Simonsson. 2009. Silvicultural models to maintain and restore natural stand structures in Swedish boreal forests. Forest Ecology and Management, 255: $2677-2688$

25. Kanowski, J., C.P. Catterall and J. Wardell. 2005. Consequences of broadscale timber plantations for biodiversity in cleared rainforest landscapes of tropical and subtropical Australia. Forest Ecology and Management, 208: 359-372.

26. Khormali, F. and S. Shamsi. 2009. Study quality and micromorphology of soil evolution in land use in loess slops East Golestan province (Case study: Ghapan). Journal of Agriculture science and natural resources, 16(3): 1-12 (In Persian).

27. Krebs, Ch. 1989. Ecological methodology. Harper Collins Publisher, 654 pp.

28. Kryshen, A.M. 2000. Dynamics of vascular plant diversity at the initial states of reforestation after clear cutting of secondary spruce stand. Proceeding of disturbance dynamics in boreal forests with the main theme: Restoration and management of biodiversity. Finland, Aug 28, 21-25.

29. Li, Y., D. Zou, B. Ren, X. Ding, H. Bian and J. Wang. 2016. Balancing effect of Larch plantations (Larix kaempferi) on understory plant diversity in a sub-tropical forest ecosystem, China. Applied Ecology and Environmental Research, 14(3): 397-407.

30. Lindenmayer, D.B. and R.J. Hobbs. 2004. Fauna conservation in Australian plantation forests: a review. Biological Conservation, 119: 151-168.

31. Ludwig, J.A. and J.F. Reynolds. 1988. Statistical ecology. Wiley-Interscience Press, New York, $337 \mathrm{pp}$.

32. Mohammadi, S., R. Rahmani and R. Arabali. 2014. Measuring throughfall and interception loss in Horizontal cypress and Turkish pine Afforestations and a natural stand of chestnut-leaved oak at Kohmian of Azadshahr, Iran. Iranian Journal of Forest, 6(3): 363-376 (In Persian).

33. Mohammadnejad kiasari, Sh., Kh. Sagheb Talebi and R. Rahmani. 2013. Comparison of Plants Diversity in Natural Forest and Afforestations (Case Study: Darabkola, Mazandaran). Journal of Wood and Forest Science and Technology, 19(4): 59-76 (In Persian).

34. Mohammadnejad kiasari, Sh., Kh. Sagheb Talebi and SH. Amini. 2017. Ecological assessment of conifers and broad-leaved plantationsin Neka, Eastern of Mazandaran (Case Study: Ghoremarez Area). Journal of Wood and Forest Science and Technology, 24(4): 173-185 (In Persian).

35. Muller-Dombois, D. and H. Ellenberg. 1974. Aims and Methods of Vegetation Ecology. John Wiley and Sons, New York, 523 pp.

36. Nagaike, T. 2002. Differences in plant species diversity between conifer (Larix kaempferi) plantations and broad leaved (Quercus crispula) secondary forests in central Japan. Forest Ecology and Management, 168: 111-123.

37. Nagaike, T. 2012. Review of plant species diversity in managed forests in Japan. International Scholarly Research Network, ISRN Forestry, ID 629523, 7 pp.

38. Nagaike, T., A. Hayashi, A. Abe and N. Arai. 2003. Differences in plant species diversity in Larix kaempferi plantations of different ages in central Japan. Forest Ecology and Management, 183: 177193.

39. Nouri, Z., J. Feghhi, Gh. Zahedi, M. Zobeiri and R. Rahmani. 2010. The Study of Shrub and Tree Species Diversity and its Application in Forest Planning (Case study: Patom District, Kheyroud Forest). Journal of Forest and Wood Products, 63(2): 201-214 (In Persian).

40. Paritsis, J. and M.A. Aizen. 2007. Effect of exotic conifer plantations on the biodiversity of understory plants, epigeal beetles and birds in Notofagus dombeyi forest. Forest Ecology and Management, 255: 1575-1583.

41. Pourbabaei, H., F. Asghari, A. Reif and R. Abedi. 2012. Effect of plantations on plant species diversity in the Darabkola, Mazandaran Province, North of Iran. Biodiversitas, 13(2): 72-78.

42. Powers, J.S., J.P. Haggar and R.F. Fisher. 1998. The effect of overstory composition on understory woody regeneration and species richness in 7-years old plantation in Costa Rica. Forest Ecology and Management, 99(3): 43-54.

43. Rostami Shahraji, T. and H. Pourbabaei. 2007. Study of vegetation in loblolly pine (Pinus taeda L.) plantations in the Aziz kian and Lakan areas, Rasht. Journal of Environmental Studies, 33(41): 85-96 (In Persian).

44. Yousefi, A., H. Jalilvand, M.R. Pourmajidian and K. Espahbodi. 2010. Understory indigenous woody species diversity in hardwood and coniferous tree plantations at Berenjestanak lowland forest in the North of Iran. Journal of Biodiversity and Conservation, 2: 273-283. 


\title{
Trends in Woody Species Diversity Across Different Age Classes of Pinus brutia and Cupressus horizontalis Plantations in Ghapan Region, East of Golestan
}

\author{
Hossein Ghorbani $^{1}$, Ramin Rahmani ${ }^{2}$, Alireza Ali Aarb ${ }^{3}$ and Asadollah Karimidoost ${ }^{4}$ \\ 1 and 3- Graduated M.Sc. Student and Assistant Professor, Forest Sciences Faculty, Gorgan University of \\ Agricultural Sciences and Natural Resources, Gorgan, Iran \\ 2- Associate Professor, Forest Sciences Faculty, Gorgan University of Agricultural Sciences and Natural Resources, \\ Gorgan, Iran, (Corresponding author: rahmani@gau.ac.ir) \\ 4- Master of Science in Research Institue, Natural Resources Research, Golestan Agricultural and Natural Resources \\ Research and Education Center, Agricultural Research and Education Organization, Gorgan, Iran

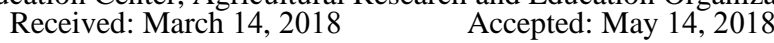

\begin{abstract}
Forest degradation influences ecosystem sustainability and reduces plant species diversity. Plantations with coniferous trees in degraded ecosystems help the native species to become established and play a major role in preserving and improving plant diversity. This study aims at measuring and comparing the woody species diversity across different age classes of Pinus brutia and Cupressus horizontalis in Ghapan region, East of Golestan. Plantations were classified in the following age classes: $<10,10-20$, and 20-30 years old. Then an area of about 5 ha were chosen for every age class and a number of 30 plots $\left(400 \mathrm{~m}^{2}\right)$ were systematic randomly selected. List of woody species and their density in each sample plot were recorded. Number of woody species was calculated using species richness, expected richness, and Margalef indices; diversity was calculated using Simpson and Shannon-Wiener indices; and evenness was calculated using Camargo and Simpson indices. Analysis of variance showed that the effects of species, age classes, and their interaction were significant. Slicing of interaction effect indicated an outstanding difference of richness, diversity, and evenness indices across the different age classes of Pinus brutia plantations. The difference of expected richness and Margalef, Simpson, and Shannon-Wiener indices were not significant across the different age classes of Cupressus horizontalis plantations. But, the increasing of species richness and evenness of Camargo and Simpson indices were significant across the age classes of $<10$ and 10-20 years old. Also, it was showed that Pinus brutia plantation is more suitable for establishment and development of woody species than Cupressus horizontalis and therefore it is recommended for future plantations at the study region.
\end{abstract}

Keywords: Diversity, Plantation, Needle leaved, Woody species, Ghapan 